\title{
Third-order many-body perturbation theory calculations for the beryllium and magnesium isoelectronic sequences
}

\author{
H. C. Ha and W. R. Johnsont \\ Department of Physics, University of Notre Dame, Notre Dame, Indiana 46556, USA \\ S. A. Blundel围 \\ Département de Recherche Fondamentale sur la Matière Condensée, CEA-Grenoble/DSM \\ 17 rue des Martyrs, F-38054 Grenoble Cedex 9, France \\ M. S. Safronova \\ Department of Physics, University of Delaware, Newark, Delaware 19716, USA
}

(Dated: July 19, 2018)

\begin{abstract}
Relativistic third-order MBPT is applied to obtain energies of ions with two valence electrons in the no virtual-pair approximation (NVPA). A total of 302 third-order Goldstone diagrams are organized into 12 one-body and 23 two-body terms. Only third-order two-body terms and diagrams are presented here, owing to the fact that the one-body terms are identical to the previously studied third-order terms in monovalent ions. Dominant classes of diagrams are identified. The model potential is a Dirac-Hartree-Fock $V^{N-2}$ potential, and B-spline basis functions in a cavity of finite radius are employed in the numerical calculations. The Breit interaction is taken into account through second order of perturbation theory and the lowest-order Lamb shift is also evaluated. Sample calculations are performed for berylliumlike ions with $Z=4-7$, and for the magnesiumlike ion P IV. The third-order energies are in excellent agreement with measurement with an accuracy at $0.2 \%$ level for the cases considered. Comparisons are made with previous second-order MBPT results and with other calculations. The third-order energy correction is shown to be significant, improving second-order correlation energies by an order of magnitude.
\end{abstract}

PACS numbers: 31.15.Ar, 31.25.Md, 31.25.Jf, 31.30.Jv

\section{INTRODUCTION}

The development of relativistic MBPT in recent decades has been motivated in part by the need for accurate theoretical amplitudes of parity non-conserving (PNC) transitions in heavy monovalent atoms such as cesium and francium. Applications of the theoretical methods developed to treat atomic PNC include support of atomic clock development, tests of QED in precision spectroscopy of highly-stripped ions, searches for time variation in the fine-structure constant, and providing precise astrophysical data.

Although nonrelativistic studies [1, 2, 3, 4, 5, 6, 7] and relativistic $\mathrm{HF}$ calculations [8, 9, 10] for divalent atoms and ions have been done for many years, only recently have relativistic many-body calculations been reported. As examples, we note that all-order relativistic MBPT calculations for transitions in berylliumlike ions with $Z=26$ and 42 were carried out by Lindroth and Hvarfner [11], while large-scale configuration-interaction (CI) calculations for transitions in C III were performed

\footnotetext{
*Electronic address: hho1@nd.edu present address: NCTS (Physics Division), 101 Sec. 2 Kuang Fu Road, Hsinchu, Taiwan, R.O.C.

${ }^{\dagger}$ Electronic address: johnson@nd.edu

¥Electronic address: steven.blundell@cea.fr

$\S$ Electronic address: msafrono@udel.edu
}

by Chen et al. [12]. Relativistic many-body calculations for magnesiumlike ions include the CI calculations of states in the $n=3$ complex by Chen and Cheng [13], and mixed CI-MBPT calculations of excitation energies in neutral Mg by Savukov and Johnson [14].

Second-order relativistic MBPT was applied to Be-like ions by Safronova et al. [15] and energies were found to be accurate at the $2 \%$ level. In this paper, we extend relativistic MBPT for divalent atoms and ions to third order. We give a detailed treatment of the two-body terms here; the one-body terms are identical to those for monovalent systems and are discussed in detail by Blundell et al. 16]. The long-range goal of the present research is to extend the relativistic singles-doubles coupled-cluster (SDCC) formalism to atoms and ions with two valence electrons. The present calculations permit us to identify and evaluate those third-order terms missing from the SDCC expansion.

\section{THEORETICAL METHOD}

The model potential for our MBPT calculation is the Dirac-Hartree-Fock $V^{N-2}$ potential. The DiracCoulomb-Breit Hamiltonian for an $N$-electron atom is $H=H_{0}+V$, where

$$
H_{0}=\sum_{i=1}^{N}\left[h_{\mathrm{D}}(i)+u\left(r_{i}\right)\right],
$$


and the Dirac Hamiltonian is

$$
h_{\mathrm{D}}=c \boldsymbol{\alpha} \cdot \mathbf{p}+\beta c^{2}+V_{\text {nuc }}(r),
$$

where $V_{\text {nuc }}$ is obtained assuming a Fermi nuclear charge distribution. All equations are in atomic units. The perturbation is

$$
V=\sum_{i<j=1}^{N}\left(\frac{1}{r_{i j}}+b_{i j}\right)-\sum_{i=1}^{N} u\left(r_{i}\right),
$$

where $b_{i j}$ is the Breit interaction

$$
b_{i j}=-\frac{1}{2 r_{i j}}\left[\boldsymbol{\alpha}_{\boldsymbol{i}} \cdot \boldsymbol{\alpha}_{\boldsymbol{j}}+\frac{\left(\boldsymbol{\alpha}_{\boldsymbol{i}} \cdot \boldsymbol{r}_{\boldsymbol{i} \boldsymbol{j}}\right)\left(\boldsymbol{\alpha}_{\boldsymbol{j}} \cdot \boldsymbol{r}_{\boldsymbol{i} \boldsymbol{j}}\right)}{r_{i j}^{2}}\right] .
$$

In the no virtual-pair approximation, the excitations are limited to positive-energy eigenstates of $H$. 17, 18, 19, 20]

The eigenstates of a divalent system having angular momentum $(J, M)$ are described by the coupled states

$$
\Phi_{J M}(v w)=\eta_{v w} \sum_{m_{v} m_{w}}\left\langle j_{v} j_{w}, m_{v} m_{w} \mid J M\right\rangle a_{v}^{\dagger} a_{w}^{\dagger}|0\rangle,
$$

where $|0\rangle$ represents the ground state of the ionic core and $\eta_{v w}$ is the normalization constant

$$
\eta_{v w}=\left\{\begin{array}{cc}
1 & \text { for } v \neq w \\
\frac{1}{\sqrt{2}} & \text { for } v=w
\end{array} .\right.
$$

Here $v$ and $w$ specify the corresponding one-electron states with quantum numbers $\left(n_{v}, l_{v}, j_{v}, m_{v}\right)$ and $\left(n_{w}, l_{w}, j_{w}, m_{w}\right)$. The model space $P$ is defined by the set of total angular-momentum states (1); the model-space projection operator is

$$
\mathbf{P}=\sum_{\substack{J M \\(v \leq w)}}\left|\Phi_{J M}(v w)\right\rangle\left\langle\Phi_{J M}(v w)\right| .
$$

The orthogonal-space operator $\mathbf{Q}$ is simply $\mathbf{1} \mathbf{- P}$.

The wave operator $\Omega$ is found by solving the generalized Bloch equation [21]

$$
\left[\Omega, H_{0}\right] \mathbf{P}=(V \Omega-\Omega \mathbf{P} V \Omega) \mathbf{P} .
$$

The effective Hamiltonian is given in terms of the wave operator

$$
H_{\mathrm{eff}}=P H_{0} P+P V \Omega P .
$$

\section{A. Effective Hamiltonian}

We first find the configuration-weight vector by diagonalizing the Hamiltonian $H=H_{\text {eff }}^{(0)}+H_{\text {eff }}^{(1)}$ using total angular-momentum eigenstates (11) as basis. Higher-order energies are obtained by operating the effective Hamiltonian of the corresponding order on the configuration-weight vector. For simplicity, matrix elements of the effective Hamiltonian are given for the uncoupled states, $|v w\rangle \equiv a_{v}^{\dagger} a_{w}^{\dagger}|0\rangle$ and $\left|v^{\prime} w^{\prime}\right\rangle$. In third order, each element consists of 12 one-body and 23 twobody terms. They represent a total of 84 one-body and 218 two-body Goldstone diagrams. The multiplications of Clebsch-Gordan coefficients and the summations over magnetic quantum numbers are carried out during angular decomposition. Only the two-body part of the third order is discussed here as the one-body part and complete second-order results are already presented in Refs. [16] 
and [15].

$$
\begin{aligned}
& Z=\sum_{a b c d} \frac{g_{c d w v} g_{a b c d} \tilde{g}_{w^{\prime} v^{\prime} a b}}{\left(\varepsilon_{a b}-\varepsilon_{v^{\prime} w^{\prime}}\right)\left(\varepsilon_{c d}-\varepsilon_{v^{\prime} w^{\prime}}\right)} \\
& S_{1}=\sum_{a b c m} \frac{g_{a c m w} \tilde{g}_{m b a c} \tilde{g}_{v^{\prime} w^{\prime} v b}}{\left(\varepsilon_{b v}-\varepsilon_{v^{\prime} w^{\prime}}\right)\left(\varepsilon_{a c v}-\varepsilon_{m v^{\prime} w^{\prime}}\right)} \\
& \times\left(1+\left[v \leftrightarrow w, v^{\prime} \leftrightarrow w^{\prime}\right]\right)+\text { c.c. } \\
& S_{2}=\sum_{a b c m} \frac{\tilde{g}_{a b w m} \tilde{g}_{m c b v} \tilde{g}_{v^{\prime} w^{\prime} c a}}{\left(\varepsilon_{a c}-\varepsilon_{v^{\prime} w^{\prime}}\right)\left(\varepsilon_{a b v}-\varepsilon_{m v^{\prime} w^{\prime}}\right)} \\
& \times\left(1+\left[v \leftrightarrow w, v^{\prime} \leftrightarrow w^{\prime}\right]\right)+\text { c.c. } \\
& S_{3}=\sum_{a b c m} \frac{g_{a b w m} \tilde{g}_{w^{\prime} c a b} \tilde{g}_{v^{\prime} m v c}}{\left(\varepsilon_{c v}-\varepsilon_{m v^{\prime}}\right)\left(\varepsilon_{a b v}-\varepsilon_{m v^{\prime} w^{\prime}}\right)} \\
& \times(1-[v \leftrightarrow w])\left(1-\left[v^{\prime} \leftrightarrow w^{\prime}\right]\right)+\text { c.c. } \\
& S_{4}=\sum_{a b c m} \frac{\tilde{g}_{a b w m} \tilde{g}_{v^{\prime} c v b} \tilde{g}_{w^{\prime} m a c}}{\left(\varepsilon_{a c}-\varepsilon_{m w^{\prime}}\right)\left(\varepsilon_{a b v}-\varepsilon_{m v^{\prime} w^{\prime}}\right)} \\
& \times(1-[v \leftrightarrow w])\left(1-\left[v^{\prime} \leftrightarrow w^{\prime}\right]\right) \\
& D_{1}=-\sum_{a b m n} \frac{g_{a b m n} \tilde{g}_{m n w b} \tilde{g}_{v^{\prime} w^{\prime} v a}}{\left(\varepsilon_{b w}-\varepsilon_{m n}\right)\left(\varepsilon_{a v}-\varepsilon_{v^{\prime} w^{\prime}}\right)} \\
& \times\left(1+\left[v \leftrightarrow w, v^{\prime} \leftrightarrow w^{\prime}\right]\right)+\text { c.c. } \\
& D_{2}=\sum_{a b m n}^{\prime} \frac{g_{a b m n} g_{m n v w} \tilde{g}_{v^{\prime} w^{\prime} a b}}{\left(\varepsilon_{a b v w}-\varepsilon_{m n v^{\prime} w^{\prime}}\right)}\left[\frac{1}{\left(\varepsilon_{v w}-\varepsilon_{m n}\right)}\right. \\
& \left.+\frac{1}{\left(\varepsilon_{a b}-\varepsilon_{v^{\prime} w^{\prime}}\right)}\right]+ \text { c.c. } \\
& D_{3}=-\sum_{a b m n}^{\prime} \frac{\tilde{g}_{a b m n} g_{w^{\prime} n a b} \tilde{g}_{v^{\prime} m v w}}{\left(\varepsilon_{a b v w}-\varepsilon_{m n v^{\prime} w^{\prime}}\right)}\left[\frac{1}{\left(\varepsilon_{a b}-\varepsilon_{n w^{\prime}}\right)}\right. \\
& \left.+\frac{1}{\left(\varepsilon_{v w}-\varepsilon_{m v^{\prime}}\right)}\right]\left(1+\left[v \leftrightarrow w, v^{\prime} \leftrightarrow w^{\prime}\right]\right)+\text { c.c. } \\
& D_{4}=\sum_{a b m n} \frac{\tilde{g}_{a b m n} \tilde{g}_{w^{\prime} n w b} \tilde{g}_{v^{\prime} m v a}}{\left(\varepsilon_{b w}-\varepsilon_{n w^{\prime}}\right)\left(\varepsilon_{a v}-\varepsilon_{m v^{\prime}}\right)}(1-[v \leftrightarrow w])+\text { c.c. } \\
& D_{5}=-\sum_{a b m n} \frac{\tilde{g}_{w^{\prime} a m n} \tilde{g}_{n b a w} \tilde{g}_{v^{\prime} m v b}}{\left(\varepsilon_{a v w}-\varepsilon_{m n v^{\prime}}\right)\left(\varepsilon_{b v}-\varepsilon_{m v^{\prime}}\right)} \\
& \times(1-[v \leftrightarrow w])\left(1-\left[v^{\prime} \leftrightarrow w^{\prime}\right]\right)+\text { c.c. } \\
& D_{6}=\sum_{a b m n} \frac{\tilde{g}_{w^{\prime} a m n} \tilde{g}_{n b v w} \tilde{g}_{v^{\prime} m a b}}{\left(\varepsilon_{a v w}-\varepsilon_{m n v^{\prime}}\right)\left(\varepsilon_{a b}-\varepsilon_{m v^{\prime}}\right)} \\
& \times\left(1+\left[v \leftrightarrow w, v^{\prime} \leftrightarrow w^{\prime}\right]\right)+\text { c.c. } \\
& D_{7}=-\sum_{a b m n} \frac{\tilde{g}_{a b m w} \tilde{g}_{w^{\prime} m b n} \tilde{g}_{v^{\prime} n v a}}{\left(\varepsilon_{a b v}-\varepsilon_{m v^{\prime} w^{\prime}}\right)\left(\varepsilon_{a v}-\varepsilon_{n v^{\prime}}\right)} \\
& \times(1-[v \leftrightarrow w])\left(1-\left[v^{\prime} \leftrightarrow w^{\prime}\right]\right)+\text { c.c. } \\
& D_{8}=\sum_{a b m n} \frac{\tilde{g}_{w^{\prime} b w n} \tilde{g}_{n a b m} \tilde{g}_{v^{\prime} m v a}}{\left(\varepsilon_{b v}-\varepsilon_{n v^{\prime}}\right)\left(\varepsilon_{a v}-\varepsilon_{m v^{\prime}}\right)} \\
& \times(1-[v \leftrightarrow w])\left(1-\left[v^{\prime} \leftrightarrow w^{\prime}\right]\right) \\
& D_{9}=-\sum_{a b m n} \frac{g_{a b w m} \tilde{g}_{v^{\prime} m v n} \tilde{g}_{w^{\prime} n a b}}{\left(\varepsilon_{a b v}-\varepsilon_{m v^{\prime} w^{\prime}}\right)\left(\varepsilon_{a b}-\varepsilon_{n w^{\prime}}\right)} \\
& \times(1-[v \leftrightarrow w])\left(1-\left[v^{\prime} \leftrightarrow w^{\prime}\right]\right)
\end{aligned}
$$

$$
\begin{aligned}
& D_{10}=-\sum_{a b m n} \frac{g_{w^{\prime} b m n} \tilde{g}_{v^{\prime} a v b} \tilde{g}_{m n w a}}{\left(\varepsilon_{b v w}-\varepsilon_{m n v^{\prime}}\right)\left(\varepsilon_{a w}-\varepsilon_{m n}\right)} \\
& \times(1-[v \leftrightarrow w])\left(1-\left[v^{\prime} \leftrightarrow w^{\prime}\right]\right) \\
& T_{1}=\sum_{a m n r}^{\prime} \frac{g_{w^{\prime} a n m} \tilde{g}_{m n a r} \tilde{g}_{v^{\prime} r v w}}{\left(\varepsilon_{a v w}-\varepsilon_{m n v^{\prime}}\right)\left(\varepsilon_{v w}-\varepsilon_{r v^{\prime}}\right)} \\
& \times\left(1+\left[v \leftrightarrow w, v^{\prime} \leftrightarrow w^{\prime}\right]\right)+\text { c.c. } \\
& T_{2}=\sum_{a m n r} \frac{g_{w^{\prime} a m n} \tilde{g}_{m n w r} \tilde{g}_{v^{\prime} r v a}}{\left(\varepsilon_{a v w}-\varepsilon_{m n v^{\prime}}\right)\left(\varepsilon_{a v}-\varepsilon_{r v^{\prime}}\right)} \\
& \times(1-[v \leftrightarrow w])\left(1-\left[v^{\prime} \leftrightarrow w^{\prime}\right]\right)+\text { c.c. } \\
& T_{3}=\sum_{a m n r}^{\prime} \frac{\tilde{g}_{w^{\prime} a n r} \tilde{g}_{v^{\prime} r m a} \tilde{g}_{m n v w}}{\left(\varepsilon_{a v w}-\varepsilon_{n r v^{\prime}}\right)\left(\varepsilon_{v w}-\varepsilon_{m n}\right)} \\
& \times\left(1+\left[v \leftrightarrow w, v^{\prime} \leftrightarrow w^{\prime}\right]\right)+\text { c.c. } \\
& T_{4}=\sum_{a m n r} \frac{\tilde{g}_{w^{\prime} a r n} \tilde{g}_{v^{\prime} n v m} \tilde{g}_{r m w a}}{\left(\varepsilon_{a v w}-\varepsilon_{n r v^{\prime}}\right)\left(\varepsilon_{a w}-\varepsilon_{m r}\right)} \\
& \times(1-[v \leftrightarrow w])\left(1-\left[v^{\prime} \leftrightarrow w^{\prime}\right]\right) \\
& Q=\sum_{m n r s}^{\prime} \frac{g_{v^{\prime} w^{\prime} r s} g_{r s m n} \tilde{g}_{m n v w}}{\left(\varepsilon_{v w}-\varepsilon_{r s}\right)\left(\varepsilon_{v w}-\varepsilon_{m n}\right)} \\
& B_{1}=-\sum_{a m n x} \frac{\tilde{g}_{w^{\prime} a m n} g_{n \max } \tilde{g}_{v^{\prime} x v w}}{\left(\varepsilon_{a v w}-\varepsilon_{m n v^{\prime}}\right)\left(\varepsilon_{a x}-\varepsilon_{m n}\right)} \\
& \times\left(1+\left[v \leftrightarrow w, v^{\prime} \leftrightarrow w^{\prime}\right]\right) \\
& B_{2}=-\sum_{a m x y} \frac{\tilde{g}_{v^{\prime} a x m} \tilde{g}_{w^{\prime} m y a} \tilde{g}_{x y v w}}{\left(\varepsilon_{a v w}-\varepsilon_{m x w^{\prime}}\right)\left(\varepsilon_{a y}-\varepsilon_{m w^{\prime}}\right)} \\
& \times\left(1+\left[v \leftrightarrow w, v^{\prime} \leftrightarrow w^{\prime}\right]\right) \\
& B_{3}=-\sum_{m n x y}^{\prime} \frac{g_{v^{\prime} w^{\prime} m n} g_{m n x y} \tilde{g}_{x y v w}}{\left(\varepsilon_{v w}-\varepsilon_{m n}\right)\left(\varepsilon_{x y}-\varepsilon_{m n}\right)} \text {, }
\end{aligned}
$$

where $g_{i j k l} \equiv\left\langle i j\left|r_{12}^{-1}\right| k l\right\rangle$ is the Coulomb matrix element defined in terms of single-electron functions as

$$
g_{i j k l}=\iint d \mathbf{r}_{\mathbf{1}} d \mathbf{r}_{\mathbf{2}} \frac{1}{\left|\mathbf{r}_{\mathbf{1}}-\mathbf{r}_{\mathbf{2}}\right|} \phi_{i}^{\dagger}\left(\mathbf{r}_{\mathbf{1}}\right) \phi_{j}^{\dagger}\left(\mathbf{r}_{\mathbf{2}}\right) \phi_{k}\left(\mathbf{r}_{\mathbf{1}}\right) \phi_{l}\left(\mathbf{r}_{\mathbf{2}}\right),
$$

and $\tilde{g}_{i j k l} \equiv g_{i j k l}-g_{i j l k}$. The notation $\varepsilon_{i j k l} \equiv \varepsilon_{i}+\varepsilon_{j}+$ $\varepsilon_{k}+\varepsilon_{l}$, etc. for the sum of single-electron eigenenergies has also been used. The third-order terms are arranged by the number of excited states in the sums over states. Zero, single, double, triple, quadruple excited-state terms are designated by the letters $Z, S, D, T, Q$. Terms associated with backwards (folded) diagrams are designated by $B$. Backwards diagrams are unique for open-shell systems and exist only in the third or higher order of MBPT. The summation indices $(a, b, c, d)$ refer to core states, $(m, n, r, s)$ refer to excited states and, in backward diagrams, indices $(x, y)$ refer to valence states. The primes above the summation signs indicate that excited states $(m, n, r, s)$ are restricted to the orthogonal space $Q$ only. This restriction applies only to the term with denominator $1 /\left(\varepsilon_{v w}-\varepsilon_{m n}\right)$ in $D_{2}$ and to the term with denominator $1 /\left(\varepsilon_{v w}-\varepsilon_{m v^{\prime}}\right)$ in $D_{3}$. The c.c. denotes complex conjugate. The conjugate diagrams are obtained by a reflection through a horizontal axis, with the initial and final states switched $(v w) \leftrightarrow\left(v^{\prime} w^{\prime}\right)$. Direct diagrams of the two-body terms are shown in Fig. 1 Technically, there are subtle changes in energy denominators 
in going from the term presented to its c.c. counterpart. These changes can be deduced by re-drawing the diagram upside-down and reading off the new denominators.

In $D_{1}$ and $D_{4}$ (but not in $D_{2}$ and $D_{3}$ ) we combined diagrams associated with double excitations that have the same numerators but different denominators using the formula

$$
\frac{1}{(A+B) A}+\frac{1}{(A+B) B}=\frac{1}{A B} .
$$

Diagrams $D_{1}-D_{4}$ are special in the sense that two orderings are possible. The ambiguous vertices are labeled by crosses in the diagrams. Many of the third-order terms in the two-body part have external exchanges and complex conjugates so each diagram illustrated in Fig. 1 has from one to eight variants. The largest fraction of computer time is spent evaluating the term $Q$ and most of the remainder is spent on repetitive evaluation of terms $D_{k}, T_{k}$ and their variants. Angular decompositions of the direct formulas are listed in the Appendix.

\section{APPLICATION AND DISCUSSION}

As a first illustration, we apply the theory described above to obtain energies of the ground state and excited states in the $n=2$ complex for Be-like ions. In Table I we give a detailed breakdown of the contributions from first-, second- and third-order perturbation theory, together with corrections from the Breit interaction and from the Lamb-shift, for excitation energies of $(2 s 2 p){ }^{3} P_{0,1,2}$ states of Be-like ions. The experimental energies are taken from the NIST database for atomic spectroscopy [22]. Energies $E^{(0+1)}$ represent the lowest-order energies including the Breit correction. Lowest-order Lamb shifts $E_{\text {Lamb }}$ are obtained following the method described in Ref. [23]. We find that the residual differences between calculated and measured energies $\Delta E$ decrease rapidly with increasing $Z$. This is expected since MBPT converges better for charged ions than for neutral atoms. In fact, for highly-charged ions, correlations are expected to decrease approximately as $Z^{2-n}$, where $n$ is the order of perturbation theory [24]. On the other hand, QED effects (Lamb shifts) become more important along an isoelectronic sequence. The results in Table [ confirm both of these trends. Results of our calculations of excitation energies of all levels in the $n=2$ complex for Be-like N (N IV) are presented in Table 1 and are seen to be in agreement with measurement to parts in $10^{4}$.

As a more involved example, we give a complete breakdown of contributions to energies of low-lying states in the $n=3$ complex for the Mg-like ion P IV in Table III For both N IV and P IV, correlations are seen to account for about $10 \%$ of the total energies. For Be-like ions, the third-order correlations are only $7-10 \%$ of the secondorder correlations. The second-order correlation energies are an order of magnitude smaller than the corresponding DHF energies. The Breit correction $B^{(2)}$, which is
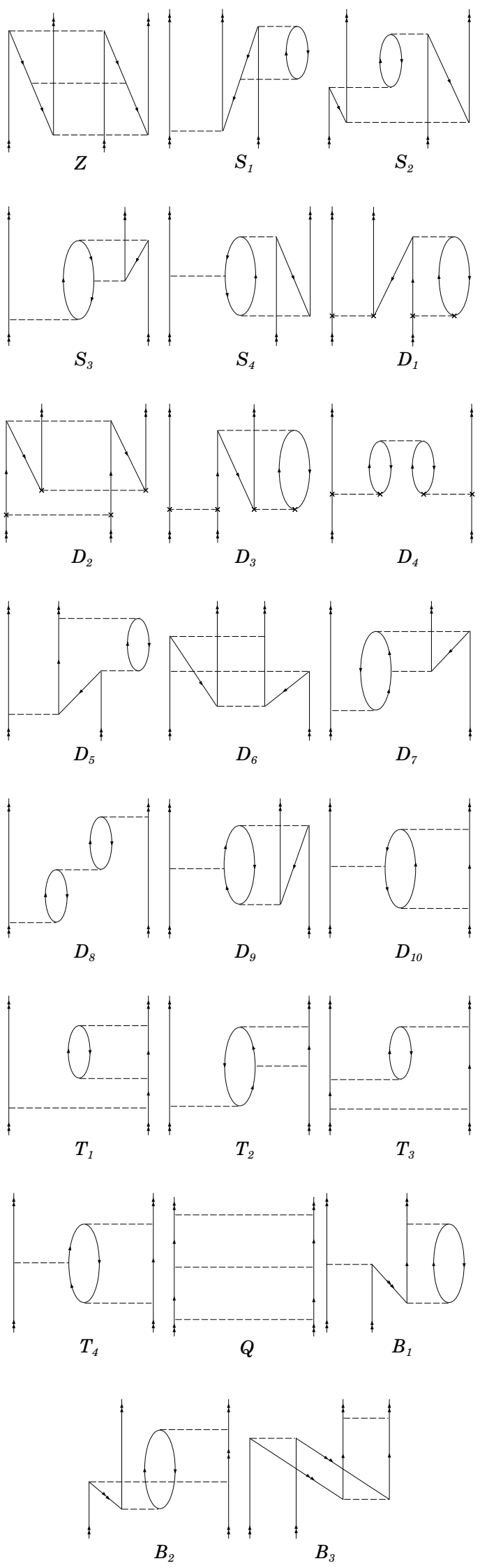

FIG. 1: Third-order Goldstone diagrams (two-body part). 
TABLE I: Comparisons of third-order energies $\left(\mathrm{cm}^{-1}\right)$ of the triplet $(2 s 2 p)^{3} P$ states of Be-like ions $Z=4-7$ with measurements are given, illustrating the rapid $\left(1 / Z^{3}\right)$ decrease of the residual correlation corrections with increasing $Z$. A breakdown of contributions to the energy from Coulomb and Breit correlation corrections and the Lamb shift is given.

\begin{tabular}{|c|c|c|c|c|}
\hline$Z$ & 4 & 5 & 6 & 7 \\
\hline \multicolumn{5}{|c|}{$(2 s 2 p)^{3} P_{0}$} \\
\hline$E^{(0+1)}$ & 23607.9 & 39116.7 & 54204.5 & 69072.2 \\
\hline$E^{(2)}$ & -3114.3 & -2583.2 & -2344.0 & -2201.5 \\
\hline$B^{(2)}$ & -1.7 & -3.7 & -6.7 & -10.5 \\
\hline$E^{(3)}$ & 473.5 & 598.0 & 412.9 & 294.9 \\
\hline$E_{\text {Lamb }}$ & -0.9 & -3.3 & -8.4 & -17.7 \\
\hline$E_{\text {tot }}$ & 20964.6 & 37124.4 & 52258.4 & 67137.3 \\
\hline$E_{\text {expt }}$ & 21978.3 & 37336.7 & 52367.1 & 67209.2 \\
\hline$\Delta E$ & -1014 & -212 & -109 & -72 \\
\hline \multicolumn{5}{|c|}{$(2 s 2 p)^{3} P_{1}$} \\
\hline$E^{(0+1)}$ & 23607.4 & 39120.2 & 54223.4 & 69127.7 \\
\hline$E^{(2)}$ & -3113.9 & -2582.2 & -2342.2 & -2198.6 \\
\hline$B^{(2)}$ & -0.6 & -1.7 & -3.3 & -5.6 \\
\hline$E^{(3)}$ & 473.4 & 597.8 & 412.7 & 294.7 \\
\hline$E_{\text {Lamb }}$ & -0.9 & -3.2 & -8.3 & -17.5 \\
\hline$E_{\text {tot }}$ & 20965.5 & 37130.9 & 52282.3 & 67200.7 \\
\hline$E_{\text {expt }}$ & 21978.9 & 37342.4 & 52390.8 & 67272.3 \\
\hline$\Delta E$ & -1013 & -212 & -109 & -72 \\
\hline \multicolumn{5}{|c|}{$(2 s 2 p)^{3} P_{2}$} \\
\hline$E^{(0+1)}$ & 23608.2 & 39132.6 & 54272.9 & 69260.8 \\
\hline$E^{(2)}$ & -3113.0 & -2580.1 & -2338.4 & -2192.6 \\
\hline$B^{(2)}$ & 0.4 & 0.5 & 0.4 & 0.0 \\
\hline$E^{(3)}$ & 473.2 & 597.4 & 412.3 & 294.1 \\
\hline$E_{\text {Lamb }}$ & -0.8 & -3.2 & -8.2 & -17.1 \\
\hline$E_{\text {tot }}$ & 20968.0 & 37147.3 & 52339.1 & 67345.2 \\
\hline$E_{\text {expt }}$ & 21981.3 & 37358.3 & 52447.1 & 67416.3 \\
\hline$\Delta E$ & -1013 & -211 & -108 & -71 \\
\hline
\end{tabular}

obtained by linearizing the second-order matrix elements in Breit interaction, is also small for such lightly-charged ions.

Our calculations for Be-like ions are able to produce results accurate to order of ten $\mathrm{cm}^{-1}$. This shows that the third-order energy correction is very important for divalent ions. By comparison, the second-order results of Safronova et al. [15] for N IV agree with experiment at the level of a few hundred $\mathrm{cm}^{-1}$. It should be noted that results from the CI+MBPT method 14] mentioned in the introduction are consistently more accurate than the present third-order results. However, the CI+MBPT calculations contain a free parameter in the energy denominators that is adjusted to give optimized energies. In contrast, third-order MBPT is completely ab initio. For the CIII ion, the large-scale CI calculations of Chen et al. 12] mentioned in the introduction also give tran- sition energies accurate to better than a hundred $\mathrm{cm}^{-1}$ on average. Those large-scale CI calculations are also $a b$ initio and have about the same accuracy as third-order MBPT for states in C III.

For the PIV ion, our results are in good agreement with experiment, the average discrepancy being several hundred $\mathrm{cm}^{-1}$. Chaudhuri et al. 25] employed an effective valence shell Hamiltonian (EVSH) to calculate energies of Mg-like ions and obtained results for P IV having a discrepancy of about a thousand $\mathrm{cm}^{-1}$, which is somewhat larger, but comparable, to the accuracy of our third-order calculations.

It is informative to analyze our results in terms of diagrams. The relative contributions of the third-order two-body terms for the ground-state energy of a typical member in the Be sequence and the ion P IV are summarized in Tables [V] and [V] Dominant classes of diagrams are $Q$ and $B_{3}$. This is understandable since $Q$ are quadruple excited-state diagrams and involve no core excitations. The quadruple excitation diagrams are entirely due to valence-valence correlation effects; they are expected to be large because of the strong repulsion of the outer valence electrons. Class $B_{3}$ are backwards diagrams, which are characteristic of open-shell systems. As shown in Fig. 11 this class is also associated solely with valence-valence correlation. The two classes $Q$ and $B_{3}$ tend to cancel each other as there is an extra phase of -1 associated with backwards diagrams. It is interesting to note that even after subtraction of the contributions from $Q$ and $B_{3}$, their difference is still larger than the contribution from any other single class of diagrams for C III.

\section{CONCLUSIONS}

The accuracy of third-order MBPT results is at $0.2 \%$ level for lightly-charged ions of both Be and $\mathrm{Mg}$ isoelectronic sequences. This level of accuracy is comparable or superior to the two ab initio methods mentioned in Sec. III A complete third-order calculation is important to understand the relative importance of different contributions to energies of divalent systems. The folded diagrams as well as the quadruple excitation diagrams are significant. The dominant role of these two classes of diagrams is attributed to the strong correlation of the two valence electrons. This conclusion is useful for workers developing combined CI-MBPT methods which include dominant third-order diagrams. It is also helpful for researchers setting up SDCC calculations as they try to classify and account for the contributions from the third-order diagrams associated with omitted triple excitations. Although one might expect a complete fourthorder calculation for divalent systems to improve the accuracy of the present calculations still further, it is unlikely that such a complex calculation will be carried out in the near future. 
TABLE II: Third-order energies $\left(\mathrm{cm}^{-1}\right)$ of states in the $n=2$ complex of the Be-like ion N IV, including corrections for the Breit interaction and the Lamb shift.

\begin{tabular}{|c|c|c|c|c|c|c|c|c|c|}
\hline & ${ }^{3} P_{0}^{o}$ & ${ }^{3} P_{1}^{o}$ & ${ }^{3} P_{2}^{o}$ & ${ }^{1} P_{1}^{o}$ & ${ }^{3} P_{0}^{e}$ & ${ }^{3} P_{1}^{e}$ & ${ }^{3} P_{2}^{e}$ & ${ }^{1} D_{2}^{e}$ & ${ }^{1} S_{0}^{e}$ \\
\hline$E_{\text {tot }}$ & 67137.3 & 67200.7 & 67345.2 & 130764.1 & 175499.4 & 175572.8 & 175699.0 & 188899.9 & 235421.9 \\
\hline$E_{\text {expt }}$ & 67209.2 & 67272.3 & 67416.3 & 130693.9 & 175535.4 & 175608.1 & 175732.9 & 188882.5 & 235369.3 \\
\hline$\Delta E$ & -72 & -72 & -71 & 70 & -36 & -35 & -34 & 17 & 53 \\
\hline
\end{tabular}

TABLE III: Comparison with measurement of theoretical energies $\left(\mathrm{cm}^{-1}\right)$ of some of the low-lying states in the $n=3$ complex of the Mg-like ion P IV, including a breakdown of contributions from Coulomb and Breit correlation corrections and the Lamb shift.

\begin{tabular}{|c|c|c|c|}
\hline & $(3 s 3 p)^{3} P_{0}$ & $(3 s 3 p)^{3} P_{1}$ & $(3 s 3 p)^{3} P_{2}$ \\
\hline$E^{(0+1)}$ & 67021.3 & 67242.9 & 67696.5 \\
\hline$E^{(2)}$ & 110.3 & 116.0 & 130.1 \\
\hline$B^{(2)}$ & -0.9 & 0.3 & 1.3 \\
\hline$E^{(3)}$ & 807.4 & 807.6 & 807.6 \\
\hline$E_{\text {Lamb }}$ & -21.1 & -20.9 & -20.5 \\
\hline$E_{\text {tot }}$ & 67917.1 & 68146.0 & 68615.0 \\
\hline$E_{\text {expt }}$ & 67918.0 & 68146.5 & 68615.2 \\
\hline \multirow[t]{2}{*}{$\Delta E$} & -0.9 & -0.5 & -0.2 \\
\hline & $(3 s 3 p){ }^{1} P_{1}$ & $\left(3 p^{2}\right){ }^{1} D_{2}$ & $\left(3 p^{2}\right)^{3} P_{0}$ \\
\hline$E^{(0+1)}$ & 120479.5 & 180554.7 & 165971.6 \\
\hline$E^{(2)}$ & -20906.0 & -61699.8 & -2027.7 \\
\hline$B^{(2)}$ & -15.8 & -8.4 & -5.2 \\
\hline$E^{(3)}$ & 6470.7 & 48769.6 & 1089.6 \\
\hline$E_{\text {Lamb }}$ & -20.7 & -43.6 & -44.0 \\
\hline$E_{\text {tot }}$ & 106007.7 & 167572.4 & 164984.3 \\
\hline$E_{\text {expt }}$ & 105190.4 & 166144.0 & 164941.4 \\
\hline \multirow[t]{2}{*}{$\Delta E$} & 817 & 1428 & 43 \\
\hline & $\left(3 p^{2}\right)^{3} P_{1}$ & $\left(3 p^{2}\right)^{3} P_{2}$ & $\left(3 p^{2}\right)^{1} S_{0}$ \\
\hline$E^{(0+1)}$ & 166200.8 & 166633.3 & 212201.4 \\
\hline$E^{(2)}$ & -2013.9 & -2008.1 & -23060.7 \\
\hline$B^{(2)}$ & -4.8 & -2.7 & -24.6 \\
\hline$E^{(3)}$ & 1087.9 & 1077.3 & 5810.7 \\
\hline$E_{\text {Lamb }}$ & -43.8 & -43.4 & -41.3 \\
\hline$E_{\text {tot }}$ & 165226.1 & 165656.5 & 194885.6 \\
\hline$E_{\text {expt }}$ & 165185.4 & 165654.0 & 194591.8 \\
\hline$\Delta E$ & 41 & 3 & 294 \\
\hline
\end{tabular}

TABLE IV: Relative contributions of third-order two-body terms for C III.

\begin{tabular}{lrlrlllllll}
\hline \hline Term & $(\%)$ & Term & $(\%)$ & Term & $(\%)$ & Term & $(\%)$ & Term & $(\%)$ \\
\hline$Z$ & 0.1 & $D_{1}$ & -0.2 & $D_{6}$ & 0.0 & $T_{1}$ & 4.2 & $B_{1}$ & -0.8 \\
$S_{1}$ & 0.1 & $D_{2}$ & 0.4 & $D_{7}$ & 0.0 & $T_{2}$ & -0.3 & $B_{2}$ & -0.1 \\
$S_{2}$ & -0.4 & $D_{3}$ & -1.6 & $D_{8}$ & 0.3 & $T_{3}$ & -0.5 & $B_{3}$ & -46.1 \\
$S_{3}$ & -0.1 & $D_{4}$ & 0.1 & $D_{9}$ & -0.1 & $T_{4}$ & 2.0 & & \\
$S_{4}$ & 0.4 & $D_{5}$ & 0.2 & $D_{10}$ & -1.0 & $Q$ & 41.2 & & \\
\hline \hline
\end{tabular}

TABLE V: Relative contributions of third-order two-body terms for P IV.

\begin{tabular}{lrlrllllll}
\hline \hline Term & $(\%)$ & Term & $(\%)$ & Term & $(\%)$ & Term & $(\%)$ & Term & $(\%)$ \\
\hline$Z$ & 0.1 & $D_{1}$ & -0.4 & $D_{6}$ & -0.1 & $T_{1}$ & 9.4 & $B_{1}$ & -3.9 \\
$S_{1}$ & 0.4 & $D_{2}$ & 0.3 & $D_{7}$ & -0.3 & $T_{2}$ & 0.1 & $B_{2}$ & -0.7 \\
$S_{2}$ & -0.3 & $D_{3}$ & -3.6 & $D_{8}$ & 0.1 & $T_{3}$ & 2.1 & $B_{3}$ & -29.2 \\
$S_{3}$ & 0.2 & $D_{4}$ & 0.6 & $D_{9}$ & -0.5 & $T_{4}$ & 10.2 & & \\
$S_{4}$ & 1.4 & $D_{5}$ & -1.1 & $D_{10}$ & -5.0 & $Q$ & 29.9 & & \\
\hline \hline
\end{tabular}

\section{Acknowledgments}

The work of H.C.H. and W.R.J. was supported in part by National Science Foundation (NSF) Grant No. PHY04-56828. The work of M.S.S. was supported in part by NSF Grant No. PHY-04-57078. 


\section{APPENDIX}

Angular decompositions of direct formulas for the third-order two-body part are presented.

$$
\begin{aligned}
& Z=\sum_{\substack{L_{1} L_{2} L_{3} \\
a b c d}} \frac{X_{L_{1}}(c d w v) X_{L_{2}}(a b c d) Z_{L_{3}}\left(w^{\prime} v^{\prime} a b\right)}{\left(\varepsilon_{a b}-\varepsilon_{v^{\prime} w^{\prime}}\right)\left(\varepsilon_{c d}-\varepsilon_{v^{\prime} w^{\prime}}\right)} \\
& \times(-1)^{J+L_{1}+L_{2}+L_{3}+j_{a}+j_{b}+j_{c}+j_{d}+j_{w^{\prime}}+j_{v}} \\
& \times\left\{\begin{array}{rrr}
J & j_{c} & j_{d} \\
L_{1} & j_{v} & j_{w}
\end{array}\right\}\left\{\begin{array}{ccc}
j_{a} & j_{b} & J \\
j_{d} & j_{c} & L_{2}
\end{array}\right\}\left\{\begin{array}{ccc}
J & j_{a} & j_{b} \\
L_{3} & j_{v^{\prime}} & j_{w^{\prime}}
\end{array}\right\} \\
& S_{1}=\frac{1}{\left[j_{w}\right]} \sum_{\substack{L L^{\prime} \\
a b c m}} \frac{X_{L}(a c m w) Z_{L}(m b a c) Z_{L^{\prime}}\left(v^{\prime} w^{\prime} v b\right)}{\left(\varepsilon_{b v}-\varepsilon_{v^{\prime} w^{\prime}}\right)\left(\varepsilon_{a c v}-\varepsilon_{m v^{\prime} w^{\prime}}\right)} \\
& \times(-1)^{J+L^{\prime}+j_{a}+j_{b}+j_{c}+j_{m}+j_{w^{\prime}}+j_{v}} \\
& \times \delta_{j_{b} j_{w}} \frac{1}{[L]}\left\{\begin{array}{rrr}
J & j_{v^{\prime}} & j_{w^{\prime}} \\
L^{\prime} & j_{b} & j_{v}
\end{array}\right\} \\
& S_{2}=\sum_{\substack{L L^{\prime} \\
a b c m}} \frac{Z_{L}(a b w m) Z_{L}(m c b v) Z_{L^{\prime}}\left(v^{\prime} w^{\prime} c a\right)}{\left(\varepsilon_{a c}-\varepsilon_{v^{\prime} w^{\prime}}\right)\left(\varepsilon_{a b v}-\varepsilon_{m v^{\prime} w^{\prime}}\right)} \\
& \times(-1)^{1+L^{\prime}+j_{a}+j_{b}+j_{c}+j_{m}+j_{w^{\prime}}+j_{v}} \\
& \times \frac{1}{[L]}\left\{\begin{array}{lll}
J & j_{a} & j_{c} \\
L & j_{v} & j_{w}
\end{array}\right\}\left\{\begin{array}{rrr}
J & j_{c} & j_{a} \\
L^{\prime} & j_{w^{\prime}} & j_{v^{\prime}}
\end{array}\right\} \\
& S_{3}=\sum_{\substack{L_{1} L_{2} L_{3} \\
a b c m}} \frac{X_{L_{1}}(a b w m) Z_{L_{2}}\left(w^{\prime} c a b\right) Z_{L_{3}}\left(v^{\prime} m v c\right)}{\left(\varepsilon_{c v}-\varepsilon_{m v^{\prime}}\right)\left(\varepsilon_{a b v}-\varepsilon_{m v^{\prime} w^{\prime}}\right)} \\
& \times(-1)^{J+j_{v}+j_{w}}\left\{\begin{array}{ccc}
L_{1} & L_{2} & L_{3} \\
j_{c} & j_{m} & j_{b}
\end{array}\right\} \\
& \times\left\{\begin{array}{ccc}
j_{w} & j_{w^{\prime}} & L_{3} \\
L_{2} & L_{1} & j_{a}
\end{array}\right\}\left\{\begin{array}{ccc}
J & j_{v^{\prime}} & j_{w^{\prime}} \\
L_{3} & j_{w} & j_{v}
\end{array}\right\} \\
& S_{4}=\sum_{\substack{L_{1} L_{2} L_{3} \\
a b c m}} \frac{Z_{L_{1}}(a b w m) Z_{L_{2}}\left(v^{\prime} c v b\right) Z_{L_{3}}\left(w^{\prime} m a c\right)}{\left(\varepsilon_{a c}-\varepsilon_{m w^{\prime}}\right)\left(\varepsilon_{a b v}-\varepsilon_{m v^{\prime} w^{\prime}}\right)} \\
& \times(-1)^{J+L_{1}+L_{2}+L_{3}+j_{v}+j_{w}}\left\{\begin{array}{ccc}
J & j_{v^{\prime}} & j_{w^{\prime}} \\
L_{2} & j_{w} & j_{v}
\end{array}\right\} \\
& \times\left\{\begin{array}{ccc}
j_{w} & j_{w^{\prime}} & L_{2} \\
L_{3} & L_{1} & j_{a}
\end{array}\right\}\left\{\begin{array}{ccc}
L_{1} & L_{2} & L_{3} \\
j_{c} & j_{m} & j_{b}
\end{array}\right\} \\
& D_{1}=-\frac{1}{\left[j_{w}\right]} \sum_{\substack{L L^{\prime} \\
a b m n}} \frac{X_{L}(a b m n) Z_{L}(m n w b) Z_{L^{\prime}}\left(v^{\prime} w^{\prime} v a\right)}{\left(\varepsilon_{b w}-\varepsilon_{m n}\right)\left(\varepsilon_{a v}-\varepsilon_{v^{\prime} w^{\prime}}\right)} \\
& \times(-1)^{J+L^{\prime}+j_{a}+j_{b}+j_{m}+j_{n}+j_{w^{\prime}}+j_{v}} \\
& \times \delta_{j_{a} j_{w}} \frac{1}{[L]}\left\{\begin{array}{ccc}
J & j_{v^{\prime}} & j_{w^{\prime}} \\
L^{\prime} & j_{a} & j_{v}
\end{array}\right\}
\end{aligned}
$$

$$
\begin{aligned}
& D_{2}=\sum_{\substack{L_{1} L_{2} L_{3} \\
a b m n}} \frac{X_{L_{1}}(a b m n) X_{L_{2}}(m n v w) Z_{L_{3}}\left(v^{\prime} w^{\prime} a b\right)}{\left(\varepsilon_{a b v w}-\varepsilon_{m n v^{\prime} w^{\prime}}\right)} \\
& \times\left[\frac{1}{\left(\varepsilon_{v w}-\varepsilon_{m n}\right)}+\frac{1}{\left(\varepsilon_{a b}-\varepsilon_{v^{\prime} w^{\prime}}\right)}\right] \\
& \times(-1)^{J+L_{1}+L_{2}+L_{3}+j_{a}+j_{b}+j_{m}+j_{n}+j_{w^{\prime}}+j_{v}} \\
& \times\left\{\begin{array}{ccc}
j_{a} & j_{b} & J \\
j_{n} & j_{m} & L_{1}
\end{array}\right\}\left\{\begin{array}{ccc}
J & j_{m} & j_{n} \\
L_{2} & j_{w} & j_{v}
\end{array}\right\}\left\{\begin{array}{rrr}
J & j_{a} & j_{b} \\
L_{3} & j_{w^{\prime}} & j_{v^{\prime}}
\end{array}\right\} \\
& D_{3}=-\frac{1}{\left[j_{w^{\prime}}\right]} \sum_{\substack{L L^{\prime} \\
a b m n}}^{\prime} \frac{Z_{L}(a b m n) X_{L}\left(w^{\prime} n a b\right) Z_{L^{\prime}}\left(v^{\prime} m v w\right)}{\left(\varepsilon_{a b v w}-\varepsilon_{m n v^{\prime} w^{\prime}}\right)} \\
& \times\left[\frac{1}{\left(\varepsilon_{a b}-\varepsilon_{n w^{\prime}}\right)}+\frac{1}{\left(\varepsilon_{v w}-\varepsilon_{m v^{\prime}}\right)}\right] \\
& \times(-1)^{J+L^{\prime}+j_{a}+j_{b}+j_{m}+j_{n}+j_{w^{\prime}}+j_{v}} \\
& \times \delta_{j_{m} j_{w^{\prime}}} \frac{1}{[L]}\left\{\begin{array}{ccc}
J & j_{v^{\prime}} & j_{m} \\
L^{\prime} & j_{w} & j_{v}
\end{array}\right\} \\
& D_{4}=\sum_{\substack{L \\
a b m n}} \frac{Z_{L}(a b m n) Z_{L}\left(w^{\prime} n w b\right) Z_{L}\left(v^{\prime} m v a\right)}{\left(\varepsilon_{b w}-\varepsilon_{n w^{\prime}}\right)\left(\varepsilon_{a v}-\varepsilon_{m v^{\prime}}\right)} \\
& \times(-1)^{J+L+j_{a}+j_{b}+j_{m}+j_{n}+j_{w^{\prime}}+j_{v}} \\
& \times \frac{1}{[L]^{2}}\left\{\begin{array}{ccc}
J & j_{w^{\prime}} & j_{v^{\prime}} \\
L & j_{v} & j_{w}
\end{array}\right\} \\
& D_{5}=-\sum_{\substack{L L^{\prime} \\
a b m n}} \frac{Z_{L}\left(w^{\prime} a m n\right) Z_{L}(n b a w) Z_{L^{\prime}}\left(v^{\prime} m v b\right)}{\left(\varepsilon_{a v w}-\varepsilon_{m n v^{\prime}}\right)\left(\varepsilon_{b v}-\varepsilon_{m v^{\prime}}\right)} \\
& \times(-1)^{J+1+L+j_{a}+j_{b}+j_{m}+j_{n}+j_{w^{\prime}}+j_{v}} \\
& \times \frac{1}{[L]}\left\{\begin{array}{rrr}
J & j_{v^{\prime}} & j_{w^{\prime}} \\
L^{\prime} & j_{w} & j_{v}
\end{array}\right\}\left\{\begin{array}{rrr}
j_{w^{\prime}} & j_{w} & L^{\prime} \\
j_{b} & j_{m} & L
\end{array}\right\} \\
& D_{6}=\sum_{\substack{L_{1} L_{2} L_{3} \\
a b m n}} \frac{Z_{L_{1}}\left(w^{\prime} a m n\right) Z_{L_{2}}(n b v w) Z_{L_{3}}\left(v^{\prime} m a b\right)}{\left(\varepsilon_{a v w}-\varepsilon_{m n v^{\prime}}\right)\left(\varepsilon_{a b}-\varepsilon_{m v^{\prime}}\right)} \\
& \times(-1)^{1+L_{1}+L_{2}+L_{3}+j_{v^{\prime}}+j_{v}} \\
& \times\left\{\begin{array}{rll}
J & j_{n} & j_{b} \\
L_{2} & j_{w} & j_{v}
\end{array}\right\}\left\{\begin{array}{ccc}
J & j_{n} & j_{b} \\
j_{w^{\prime}} & L_{1} & j_{m} \\
j_{v^{\prime}} & j_{a} & L_{3}
\end{array}\right\} \\
& D_{7}=-\sum_{\substack{L_{1} L_{2} L_{3} \\
a b m n}} \frac{Z_{L_{1}}(a b m w) Z_{L_{2}}\left(w^{\prime} m b n\right) Z_{L_{3}}\left(v^{\prime} n v a\right)}{\left(\varepsilon_{a b v}-\varepsilon_{m v^{\prime} w^{\prime}}\right)\left(\varepsilon_{a v}-\varepsilon_{n v^{\prime}}\right)} \\
& \times(-1)^{J+L_{1}+L_{2}+L_{3}+j_{v}+j_{w}}\left\{\begin{array}{ccc}
L_{1} & L_{2} & L_{3} \\
j_{n} & j_{a} & j_{m}
\end{array}\right\} \\
& \times\left\{\begin{array}{ccc}
j_{w} & j_{w^{\prime}} & L_{3} \\
L_{2} & L_{1} & j_{b}
\end{array}\right\}\left\{\begin{array}{ccc}
J & j_{v^{\prime}} & j_{w^{\prime}} \\
L_{3} & j_{w} & j_{v}
\end{array}\right\} \\
& D_{8}=\sum_{\substack{L \\
a b m n}} \frac{Z_{L}\left(w^{\prime} b w n\right) Z_{L}(n a b m) Z_{L}\left(v^{\prime} m v a\right)}{\left(\varepsilon_{b v}-\varepsilon_{n v^{\prime}}\right)\left(\varepsilon_{a v}-\varepsilon_{m v^{\prime}}\right)} \\
& \times(-1)^{J+L+j_{a}+j_{b}+j_{m}+j_{n}+j_{w^{\prime}}+j_{v}} \\
& \times \frac{1}{[L]^{2}}\left\{\begin{array}{rrr}
J & j_{w^{\prime}} & j_{v^{\prime}} \\
L & j_{v} & j_{w}
\end{array}\right\}
\end{aligned}
$$




$$
\begin{aligned}
& D_{9}=-\sum_{\substack{L_{1} L_{2} L_{3} \\
a b m n}} \frac{X_{L_{1}}(a b w m) Z_{L_{2}}\left(v^{\prime} m v n\right) Z_{L_{3}}\left(w^{\prime} n a b\right)}{\left(\varepsilon_{a b v}-\varepsilon_{m v^{\prime} w^{\prime}}\right)\left(\varepsilon_{a b}-\varepsilon_{n w^{\prime}}\right)} \\
& \times(-1)^{J+j_{v}+j_{w}}\left\{\begin{array}{ccc}
L_{1} & L_{2} & L_{3} \\
j_{n} & j_{b} & j_{m}
\end{array}\right\} \\
& \times\left\{\begin{array}{ccc}
j_{w} & j_{w^{\prime}} & L_{2} \\
L_{3} & L_{1} & j_{a}
\end{array}\right\}\left\{\begin{array}{ccc}
J & j_{v^{\prime}} & j_{w^{\prime}} \\
L_{2} & j_{w} & j_{v}
\end{array}\right\} \\
& D_{10}=-\sum_{\substack{L_{1} L_{2} L_{3} \\
a b m n}} \frac{X_{L_{1}}\left(w^{\prime} b m n\right) Z_{L_{2}}\left(v^{\prime} a v b\right) Z_{L_{3}}(m n w a)}{\left(\varepsilon_{b v w}-\varepsilon_{m n v^{\prime}}\right)\left(\varepsilon_{a w}-\varepsilon_{m n}\right)} \\
& \times(-1)^{J+j_{v}+j_{w}}\left\{\begin{array}{ccc}
L_{1} & L_{2} & L_{3} \\
j_{a} & j_{n} & j_{b}
\end{array}\right\} \\
& \times\left\{\begin{array}{ccc}
j_{w^{\prime}} & j_{w} & L_{2} \\
L_{3} & L_{1} & j_{m}
\end{array}\right\}\left\{\begin{array}{ccc}
J & j_{v^{\prime}} & j_{w^{\prime}} \\
L_{2} & j_{w} & j_{v}
\end{array}\right\} \\
& T_{1}=\frac{1}{\left[j_{w^{\prime}}\right]} \sum_{\substack{L L L^{\prime} \\
a m n r}}^{\prime} \frac{X_{L}\left(w^{\prime} a n m\right) Z_{L}(m n a r) Z_{L^{\prime}}\left(v^{\prime} r v w\right)}{\left(\varepsilon_{a v w}-\varepsilon_{m n v^{\prime}}\right)\left(\varepsilon_{v w}-\varepsilon_{r v^{\prime}}\right)} \\
& \times(-1)^{J+1+L^{\prime}+j_{a}+j_{m}+j_{n}+j_{v}} \\
& B_{2}=-\sum_{\substack{L L^{\prime} \\
a m x y}} \frac{Z_{L}\left(v^{\prime} a x m\right) Z_{L}\left(w^{\prime} m y a\right) Z_{L^{\prime}}(x y v w)}{\left(\varepsilon_{a v w}-\varepsilon_{m x w^{\prime}}\right)\left(\varepsilon_{a y}-\varepsilon_{m w^{\prime}}\right)} \\
& \times(-1)^{1+L^{\prime}+j_{a}+j_{m}+j_{x}+j_{y}+j_{w^{\prime}}+j_{v}} \\
& \times \frac{1}{[L]}\left\{\begin{array}{ccc}
J & j_{x} & j_{y} \\
L & j_{w^{\prime}} & j_{v^{\prime}}
\end{array}\right\}\left\{\begin{array}{ccc}
J & j_{x} & j_{y} \\
L^{\prime} & j_{w} & j_{v}
\end{array}\right\} \\
& B_{3}=-\sum_{\substack{L_{1} L_{2} L_{3} \\
m n x y}} \frac{X_{L_{1}}\left(v^{\prime} w^{\prime} m n\right) X_{L_{2}}(m n x y) Z_{L_{3}}(x y v w)}{\left(\varepsilon_{v w}-\varepsilon_{m n}\right)\left(\varepsilon_{x y}-\varepsilon_{m n}\right)} \\
& \times(-1)^{J+L_{1}+L_{2}+L_{3}+j_{m}+j_{n}+j_{x}+j_{y}+j_{w^{\prime}}+j_{v}} \\
& \times\left\{\begin{array}{ccc}
J & j_{m} & j_{n} \\
L_{1} & j_{w^{\prime}} & j_{v^{\prime}}
\end{array}\right\}\left\{\begin{array}{ccc}
j_{m} & j_{n} & J \\
j_{y} & j_{x} & L_{2}
\end{array}\right\}\left\{\begin{array}{ccc}
J & j_{x} & j_{y} \\
L_{3} & j_{w} & j_{v}
\end{array}\right\} .
\end{aligned}
$$$$
\times \delta_{j_{r} j_{w^{\prime}}} \frac{1}{[L]}\left\{\begin{array}{ccc}
J & j_{v^{\prime}} & j_{w^{\prime}} \\
L^{\prime} & j_{w} & j_{v}
\end{array}\right\}
$$$$
T_{2}=\sum_{\substack{L_{1} L_{2} L_{3} \\ a m n n}} \frac{X_{L_{1}}\left(w^{\prime} a m n\right) Z_{L_{2}}(m n w r) Z_{L_{3}}\left(v^{\prime} r v a\right)}{\left(\varepsilon_{a v w}-\varepsilon_{m n v^{\prime}}\right)\left(\varepsilon_{a v}-\varepsilon_{r v^{\prime}}\right)}
$$$$
\times(-1)^{J+j_{v}+j_{w}}\left\{\begin{array}{ccc}
L_{1} & L_{2} & L_{3} \\
j_{r} & j_{a} & j_{n}
\end{array}\right\}
$$$$
\times\left\{\begin{array}{ccc}
j_{w^{\prime}} & j_{w} & L_{3} \\
L_{2} & L_{1} & j_{m}
\end{array}\right\}\left\{\begin{array}{ccc}
J & j_{v^{\prime}} & j_{w^{\prime}} \\
L_{3} & j_{w} & j_{v}
\end{array}\right\}
$$$$
T_{3}=\sum_{\substack{L L^{\prime} \\ a m n r}}^{\prime} \frac{Z_{L}\left(w^{\prime} a n r\right) Z_{L}\left(v^{\prime} r m a\right) Z_{L^{\prime}}(m n v w)}{\left(\varepsilon_{a v w}-\varepsilon_{n r v^{\prime}}\right)\left(\varepsilon_{v w}-\varepsilon_{m n}\right)}
$$$$
\times(-1)^{1+L^{\prime}+j_{a}+j_{m}+j_{n}+j_{r}+j_{w^{\prime}}+j_{v}}
$$$$
\times \frac{1}{[L]}\left\{\begin{array}{ccc}
J & j_{n} & j_{m} \\
L & j_{v^{\prime}} & j_{w^{\prime}}
\end{array}\right\}\left\{\begin{array}{ccc}
J & j_{m} & j_{n} \\
L^{\prime} & j_{w} & j_{v}
\end{array}\right\}
$$$$
T_{4}=\sum_{\substack{L_{1} L_{2} L_{3} \\ a m n r}} \frac{Z_{L_{1}}\left(w^{\prime} a r n\right) Z_{L_{2}}\left(v^{\prime} n v m\right) Z_{L_{3}}(r m w a)}{\left(\varepsilon_{a v w}-\varepsilon_{n r v^{\prime}}\right)\left(\varepsilon_{a w}-\varepsilon_{m r}\right)}
$$$$
\times(-1)^{J+L_{1}+L_{2}+L_{3}+j_{v}+j_{w}}\left\{\begin{array}{ccc}
L_{1} & L_{2} & L_{3} \\
j_{m} & j_{a} & j_{n}
\end{array}\right\}
$$$$
\times\left\{\begin{array}{ccc}
j_{w^{\prime}} & j_{w} & L_{2} \\
L_{3} & L_{1} & j_{r}
\end{array}\right\}\left\{\begin{array}{ccc}
J & j_{v^{\prime}} & j_{w^{\prime}} \\
L_{2} & j_{w} & j_{v}
\end{array}\right\}
$$$$
Q=\sum_{\substack{L_{1} L_{2} L_{3} \\ m n n r s}} \frac{X_{L_{1}}\left(v^{\prime} w^{\prime} r s\right) X_{L_{2}}(r s m n) Z_{L_{3}}(m n v w)}{\left(\varepsilon_{v w}-\varepsilon_{r s}\right)\left(\varepsilon_{v w}-\varepsilon_{m n}\right)}
$$$$
\times(-1)^{J+L_{1}+L_{2}+L_{3}+j_{m}+j_{n}+j_{r}+j_{s}+j_{w^{\prime}}+j_{v}}
$$$$
\times\left\{\begin{array}{ccc}
J & j_{r} & j_{s} \\
L_{1} & j_{w^{\prime}} & j_{v^{\prime}}
\end{array}\right\}\left\{\begin{array}{ccc}
j_{r} & j_{s} & J \\
j_{n} & j_{m} & L_{2}
\end{array}\right\}\left\{\begin{array}{ccc}
J & j_{m} & j_{n} \\
L_{3} & j_{w} & j_{v}
\end{array}\right\}
$$

The effective interaction strength

$$
X_{L}(i j k l)=(-1)^{L}\left\langle i\left\|C^{L}\right\| k\right\rangle\left\langle j\left\|C^{L}\right\| l\right\rangle R_{L}(i j k l),
$$

is independent of magnetic quantum numbers. The reduced matrix element of the $\mathbf{C}^{L}$ tensor is

$$
\left\langle i\left\|C^{L}\right\| k\right\rangle=(-1)^{j_{i}+\frac{1}{2}} \sqrt{\left[j_{i}\right]\left[j_{k}\right]}\left(\begin{array}{ccc}
j_{i} & j_{k} & L \\
-\frac{1}{2} & \frac{1}{2} & 0
\end{array}\right) \Pi^{e}\left(l_{i}, l_{k}, L\right),
$$

where $\left[j_{i}\right] \equiv 2 j_{i}+1$ is the occupation number of shell $i$, and

$$
\Pi^{e}\left(l_{i}, l_{k}, L\right)=\left\{\begin{array}{ll}
1 & \text { if } l_{i}+l_{k}+L \text { is even } \\
0 & \text { if } l_{i}+l_{k}+L \text { is odd }
\end{array} .\right.
$$

The Slater integral $R_{L}(i j k l)$ is

$$
\begin{aligned}
R_{L}(i j k l)= & \int_{0}^{\infty} \int_{0}^{\infty} d r_{1} d r_{2} \frac{r_{<}^{L}}{r_{>}^{L+1}} \\
& {\left[P_{i}\left(r_{1}\right) P_{k}\left(r_{1}\right)+Q_{i}\left(r_{1}\right) Q_{k}\left(r_{1}\right)\right] } \\
& \times\left[P_{j}\left(r_{2}\right) P_{l}\left(r_{2}\right)+Q_{j}\left(r_{2}\right) Q_{l}\left(r_{2}\right)\right] .
\end{aligned}
$$

The quantity $Z_{L}(i j k l)$ is defined by

$$
Z_{L}(i j k l) \equiv X_{L}(i j k l)+[L] \sum_{L^{\prime}}\left\{\begin{array}{ccc}
j_{j} & j_{l} & L \\
j_{i} & j_{k} & L^{\prime}
\end{array}\right\} X_{L^{\prime}}(i j l k) .
$$

[1] A. Ivanova, U. Safronova, and V. Tolmachev, Litov. Phys. Sb. 7, 571 (1967).
[2] U. Safronova and A. Ivanova, Opt. Spectrosc 27, 193 (1969). 
[3] E. Ivanova and U. Safronova, J. Phys. B 8, 1591 (1975).

[4] T. N. Chang, Phys. Rev. A 39, 4946 (1989).

[5] T. N. Chang, Phys. Rev. A 34, 4550 (1986).

[6] C. Fischer, M. Godefroid, and J. Olsen, J. Phys. B 30, 1163 (1997).

[7] F. Galvez, E. Buendia, and A. Sarsa, J. Chem. Phys. 118, 6858 (2003).

[8] K. Cheng, Y. Kim, and J. Desclaux, At. Data Nucl. Data Tables 24, 111 (1979).

[9] A. Ynnerman and C. Froese-Fisher, Phys. Rev. A 51, 2020 (1995).

[10] P. Jonsson and C. Fischer, J. Phys. B 30, 5861 (1997).

[11] E. Lindroth and J. Hvarfner, Phys. Rev. A 45, 2771 (1992).

[12] M. Chen, K. Cheng, and W. Johnson, Phys. Rev. A 64, 042507 (2001).

[13] M. Chen and K. Cheng, Phys. Rev. A 55, 3440 (1997).

[14] I. Savukov and W. Johnson, Phys. Rev. A 65, 042503 (2002).
[15] M. Safronova, W. Johnson, and U. Safronova, Phys. Rev. A 53, 4036 (1996).

[16] S. Blundell, W. Johnson, and J. Sapirstein, Phys. Rev. A 42, 3751 (1990).

[17] J. Sucher, Phys. Rev. A 22, 348 (1980).

[18] M. Mittleman, Phys. Rev. A 4, 893 (1971).

[19] M. Mittleman, Phys. Rev. A 5, 2395 (1972).

[20] M. Mittleman, Phys. Rev. A 24, 1167 (1981).

[21] I. Lindgren and J. Morrison, Atomic Many-Body Theory (Springer-Verlag, Berlin, 1986), 2nd ed.

[22] Tech. Rep., National Institute of Standards and Technology (2006), uRL: http://physics.nist.gov/PhysRefData/ASD/index.html

[23] K. Cheng, W. Johnson, and J. Sapirstein, Phys. Rev. A 47, 1817 (1993).

[24] J. Sapirstein, Phys. Scr. 46, 52 (1993).

[25] R. Chaudhuri, B. Das, and K. Freed, J. Chem. Phys. 108, 2556 (1998). 